\title{
The Sensory Cortical Representation of the Human Penis: Revisiting Somatotopy in the Male Homunculus
}

\author{
Christian A. Kell, ${ }^{1,2}$ Katharina von Kriegstein, ${ }^{1,2}$ Alexander Rösler, ${ }^{3}$ Andreas Kleinschmidt, ${ }^{1,2}$ and Helmut Laufs ${ }^{1,2}$ \\ ${ }^{1}$ Brain Imaging Center and ${ }^{2}$ Department of Neurology, Johann Wolfgang Goethe University, 60590 Frankfurt, Germany, and ${ }^{3}$ Albertinen Haus Geriatric \\ Center, Department of Geriatrics and Gerontology, University of Hamburg, 22459 Hamburg, Germany
}

\begin{abstract}
Pioneering mapping studies of the human cortex have established the notion of somatotopy in sensory representation, which transpired into Penfield and Rasmussen's famous sensory homunculus diagram. However, regarding the primary cortical representation of the genitals, classical and modern findings appear to be at odds with the principle of somatotopy, often assigning it to the cortex on the mesial wall. Using functional neuroimaging, we established a mediolateral sequence of somatosensory foot, penis, and lower abdominal wall representation on the contralateral postcentral gyrus in primary sensory cortex and a bilateral secondary somatosensory representation in the parietal operculum.
\end{abstract}

Key words: somatosensory; sequence; genital; primary sensory; secondary sensory; postcentral gyrus; MRI; functional; brain

\section{Introduction}

Textbooks of neurology and neuroscience have engraved into the visual memories of generations of doctors and researchers the so-called homunculus diagrams. They were introduced by Penfield and collaborators as a means of summarizing the functional organization of the primary motor and sensory cortices that they had studied via electrical stimulation during open brain surgery (Penfield and Rasmussen, 1950). Their drawings in essence confirmed older charts but displaced them in popularity because, at a single glance, they compellingly illustrated somatotopy as a key principle in the layout of these cortices. However, on closer inspection of the somatosensory homunculus, one inevitably notes a violation of somatotopic continuity regarding the localization of the genitals. Why should they be represented below the toes in the mesial wall (Foerster, 1936)?

A detailed reexamination of the classical literature on this issue yields conflicting findings. In lesion studies performed at the beginning of the last century, Pfeifer (1920) found a genital representation between that of the leg and the trunk within the postcentral gyrus. In the 1930s, Foerster (1936) reported a genital representation on the mesial aspect of the hemisphere in the paracentral lobule, just below the representation of the toes, the latter also extending onto the convexity. The most comprehensive report was published by Penfield and Rasmussen in the 1950s. Like others, they concluded that the systematic somatotopic sequence of the sensory foci for different body parts was constant but that their individual size was variable. Remarkably, in only three of their 400 patients was genital sensation elicited by

Received Feb. 22, 2005; revised May 4, 2005; accepted May 5, 2005.

The Brain Imaging Center is funded by the German Education and Research Ministry (DLR 01G00203). We thank our volunteers, the Volkswagen Foundation for financial support, and T. Wallenhorst for technical assistance.

Correspondence should be addressed to Christian A. Kell, Brain Imaging Center, University of Frankfurt, Schleusenweg 2-16, 60528 Frankfurt, Germany. E-mail: c.kell@em.uni-frankfurt.de.

D01:10.1523/JNEUROSCI.0712-05.2005

Copyright @ 2005 Society for Neuroscience $\quad$ 0270-6474/05/255984-04\$15.00/0 electrical stimulation of the cortex adjacent to the central fissure, $1 \mathrm{~cm}$ posterior to the representation of the upper leg and lower trunk (Penfield and Rasmussen, 1950). Interestingly, the authors were not able to evoke any genital sensation by stimulation of the mesial surface of the postcentral gyrus, but this finding was not incorporated into the drawing of the sensory homunculus. The isolated location of the genital of the homunculus previously bothered Penfield's contemporaries; his scholar J. Kershman, known for his wit, pitied the creature, "His happiness founded on things near his toes; That need not always be numb" (Feinsod, 2005).

Present day neuroscience studies have recorded cerebral responses evoked by dorsal penile nerve stimulation via magnetoencephalography and electrocorticography but again have produced conflicting findings, assigning the penile representation either to the mesial wall (Allison et al., 1996; Nakagawa et al., 1998; Makela et al., 2003) or also to the convexity of the contralateral hemisphere (Bradley et al., 1998). Using functional magnetic resonance imaging (fMRI) as a high-resolution mapping technique, we therefore reexamined the cortical representation of the penis in the human somatosensory cortex and studied its topographical relationship to that of adjacent body parts.

\section{Materials and Methods}

Eight healthy male volunteers [six right-handed, two left-handed (subjects 1 and 4); 27-35 years of age; mean age, 30; informed consent] without neurological or urological illness were scanned during resting wakefulness with the use of a Siemens (Erlangen, Germany) Allegra 3T magnetic resonance scanner with a standard head coil and gradient booster. Each of three (subjects 1-4) or four (subjects 5-8) consecutive 10 min sessions yielded $240 \mathrm{~T} 2^{\star}$-weighted echoplanar image volumes covering the entire cerebrum [voxel size $3 \times 3 \times 3 \mathrm{~mm}^{3}$; 26 oblique transverse slices; $1 \mathrm{~mm}$ gap; repetition time (TR), $2500 \mathrm{~ms}$; echo time (TE), $30 \mathrm{~ms}$. Anatomical volume scans were obtained by using a magnetization-prepared rapid-acquisition gradient echo sequence (160 slices; $1 \mathrm{slab}$; TR, $2300 \mathrm{~ms}$ ). 
In contrast to previous studies that applied electrophysiological sensory stimulation techniques (Allison et al., 1996; Bradley et al., 1998; Nakagawa et al., 1998; Makela et al., 2003), the stimulus "touch" was chosen to present a physiological stimulus; with an ultra-sensitive toothbrush (Dr. Best Flex Sensitive; GlaxoSmithKline Consumer Healthcare, Buehl, Germany) we brushed the medial aspect of the left hallux (dermatome L5), the left penile shaft (L1), the left prepuce (glans in subject 5; L1), and the left lateral abdominal wall (Th11; subjects 5-8) in craniocaudal direction at $\sim 2 \mathrm{~Hz}$. The latter condition was added to establish the relationship of the penile representation to sensory foci identified more laterally. The proximal and distal portions of the penis were stimulated to elucidate the extent of the cortical penile representation (Bradley et al., 1998). To rule out the possibility that dynamic stimuli such as brushing revealed different central representations compared with pure touch, we performed a control task in subject 8 in which the skin in the same areas as the ones being brushed was solely touched at the same frequency. This mode of stimulation did not reveal different fMRI responses. Neither pain nor discomfort was reported, except by subject 4 , who reported mild pain with stimulation of the prepuce. No sexual responses or feelings were elicited.

Statistical parametric mapping 2 (SPM2; http://www.fil.ion.ucl.ac.uk/ $\mathrm{spm} /$ ) was used to perform standard spatial preprocessing (realignment, normalization, and smoothing with a $5 \mathrm{~mm}$ full-width at half-maximum isotropic Gaussian kernel). Anatomical scans were normalized to the T1 template provided by SPM2. Then the data were analyzed in the framework of the general linear model. The four conditions of interest $(10 \mathrm{~s}$ of stimulation alternating with $20 \mathrm{~s}$ of rest in pseudorandomized order: hallux, penile shaft, prepuce, and abdominal wall) of the four runs were modeled by using a boxcar function convolved with a canonical hemodynamic response function. Realignment parameters were entered into the model as effects of no interest to account for confounding motion effects. Because we expected activations contralaterally in the primary sensory cortex and bilaterally in the secondary sensory cortex, we created regions of interest as templates that covered either the medial portion of the right hemispheric central region including the mesial wall (Rolandic template) (see Fig. 1) or both the left and right operculum (opercular template) (see Fig. 2). The single-subject analyses were thresholded at $p<0.05$, corrected for multiple comparisons within the template volumes. Individual activations were overlaid onto coregistered anatomical scans. To extend the validity of our findings beyond the subjects that were studied, we performed a random effects group analysis. Because the location expected in this approach is hypothesis-driven, the results were thresholded at $p<0.001$, uncorrected. Coordinates of activations were given in the standard stereotactic space (Talairach and Tournoux, 1988); values were obtained by using a nonlinear transformation to convert coordinates from Montreal Neurological Institute space to Talairach space (mni2tal; http://www.mrc-cbu.cam.ac.uk/Imaging/Common/ mnispace.shtml).

Brodmann areas corresponding to the activations were identified by using probability maps of the anatomy tool for SPM2 (Eickhoff et al., 2005).

\section{Results}

Brushing the skin of the penis as well as of the big toe and the lower abdominal wall evoked significant focal activations in the contralateral primary somatosensory cortex (Fig. 1, Table 1). In addition, distributed bilateral activation clusters were found in opercular secondary somatosensory cortices (Fig. 2, Table 1). No other brain regions showed significant activations. The fMRI responses to toe stimulation were located in the contralateral postcentral gyrus at the medial edge of the convexity but did not descend along the mesial wall. The primary sensory representation of the penile tip and the proximal penile shaft colocalized to an area $\sim 1 \mathrm{~cm}$ lateral of the toe representation. The overall robustness of this localization in terms of normalized stereotactic coordinates (and thus brain shape) could be confirmed in a random effects analysis (Fig. 2, Table 1). With a probability of $60 \%$,

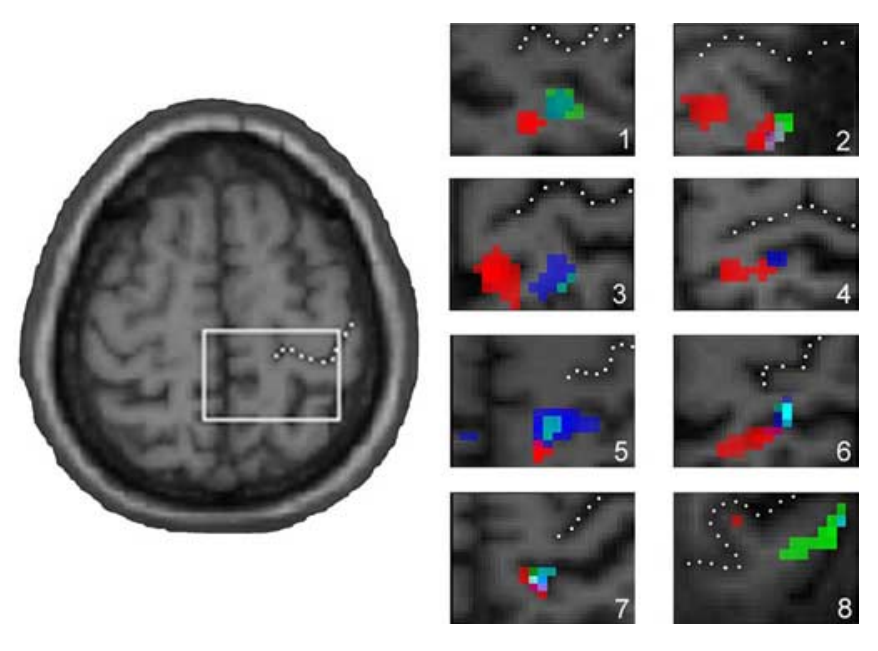

Figure 1. The sensory focus of the penis lies lateral to that of the toe. Significant fMRI activations (see Materials and Methods) in the right primary somatosensory cortex after stimulation of the contralateral hallux (red), prepuce or glans (green), penile shaft (blue), and lower abdominal wall (cyan in subjects 5-8) are overlaid onto each individual's anatomical scan. The square depicts the Rolandic template for analysis of primary sensory cortex, and dotted lines mark the central sulcus.

it can be assumed that the activations lay entirely within either Brodmann area 1 or Brodmann area $3 \mathrm{~b}$. The primary penile representation overlapped with that of the lower abdominal wall, which had a slightly more lateral center of gravity. Although the exact location of sensory foci in the primary sensory cortex varied among individuals, the sequential somatotopic representation of the lower half of the body was found in each and every subject (Fig. 1, Table 1).

In the secondary somatosensory cortex, the stimulation of the toe resulted in a bilateral activation of two cortical areas within the parietal operculum, located on the surface of the upper bank of the lateral sulcus. The rostral activation lay in the vicinity of the lateral margin of the postcentral gyrus, whereas the caudal activation was situated in the caudal parietal operculum neighboring the supramarginal gyrus (Fig. 2). Stimulation of the penis gave rise to a more lateralized pattern of opercular activation with contralateral emphasis. Interestingly, in the secondary somatosensory cortex, the hemodynamic responses that followed penile stimulation did not overlap exactly with the toe representation but lay slightly more anterolaterally.

\section{Discussion}

The primary sensory representation of the penis

Our finding that the primary sensory representation of the human penis lies on the convexity of the postcentral gyrus in an area overlapping with the representation of the lower abdominal wall confirms previous data (Pfeifer, 1920; Penfield and Rasmussen, 1950), which were overruled when the homunculus was drawn. Several other electrophysiological studies have reported a penile representation on the mesial surface of the parietal lobe (Foerster, 1936; Allison et al., 1996; Nakagawa et al., 1998; Makela et al., 2003). Erroneously melting these two into one has in the past led to grotesquely exaggerated maps of penile representation that in one proposal extended from the mesial wall to the lateral surface of the postcentral gyrus (Bradley et al., 1998). It is conceivable that the demonstration of sensory foci along the mesial wall reported in these studies can be attributed to the use of artificial electrical stimuli compared with the "physiological" stimulation used in this study, because electrical stimulation is known to 
Table 1. Individual and group activation foci in response to somatosensory stimulation

\begin{tabular}{|c|c|c|c|c|c|c|c|c|}
\hline \multirow[b]{2}{*}{ Single subject } & \multicolumn{2}{|l|}{ Toe } & \multicolumn{2}{|l|}{ Prepuce } & \multicolumn{2}{|l|}{ Penile shaft } & \multicolumn{2}{|l|}{ Lower abdominal wall } \\
\hline & Center of gravity $(x, y, z)$ & $t$ value & Center of gravity $(x, y, z)$ & $t$ value & Center of gravity $(x, y, z)$ & $t$ value & Center of gravity $(x, y, z)$ & $t$ value \\
\hline 1 & $22,-35,68$ & 4.07 & $28,-31,70$ & 6.76 & $28,-31,70$ & 6.12 & & \\
\hline 2 & $14,-33,74$ & 5.12 & $28,-39,72$ & 5.6 & $28,-39,70$ & 6.29 & & \\
\hline 3 & $6,-35,68$ & 7.86 & $22,-39,70$ & 5.18 & $18,-37,70$ & 6.69 & & \\
\hline 4 & $14,-33,75$ & 7.14 & - & - & $20,-34,68$ & 4.01 & & \\
\hline 5 & $10,-46,61$ & 6.03 & $12,-40,59$ & 5.65 & $14,-40,57$ & 6.2 & $14,-40,59$ & 8.26 \\
\hline 6 & $20,-37,74$ & 5.5 & $26,-31,73$ & 5.53 & $26,-31,73$ & 8.11 & $28,-29,71$ & 8.3 \\
\hline 7 & $10,-41,70$ & 7.9 & $12,-40,65$ & $3.53^{*}$ & $12,-40,61$ & 4.81 & $14,-38,61$ & 7.02 \\
\hline \multirow[t]{2}{*}{8} & $6,-37,74$ & 6.07 & $32,-41,70$ & 4.39 & $34,-39,70$ & $3.2^{*}$ & $38,-37,68$ & $3.25^{*}$ \\
\hline & \multicolumn{2}{|l|}{ Toe } & \multicolumn{2}{|l|}{ Prepuce } & \multicolumn{2}{|l|}{ Penile shaft } & & \\
\hline \multirow[t]{6}{*}{ Group } & Center of gravity $(x, y, z)$ & $t$ value & Center of gravity $(x, y, z)$ & $t$ value & Center of gravity $(x, y, z)$ & $t$ value & Region & \\
\hline & $12,-39,72$ & 7.38 & $24,-33,72$ & $3.43^{* *}$ & $24,-35,68$ & $3.43^{* *}$ & SI contralateral & \\
\hline & $50,-24,25$ & 11.3 & $61,-21,14$ & 8.02 & $61,-21,16$ & 5.45 & OP1 contralateral & \\
\hline & $55,2,2$ & 5.91 & $53,0,0$ & 7.62 & $63,-11,12$ & $3.47^{* *}$ & OP4 contralateral & \\
\hline & $-53,-24,21$ & 5.75 & $-63,-15,15$ & 4.77 & $-63,-22,34$ & $4.42^{* *}$ & OP1 ipsilateral & \\
\hline & $-50,-4,2$ & 10.83 & $-55,6,2$ & $3.86^{* *}$ & $-51,-4,4$ & 5.15 & OP4 ipsilateral & \\
\hline
\end{tabular}

Coordinates indicate the individual local maxima in the $x$ (mediolateral, with positive values indicating right-hemispheric), $y$ (rostrocaudal, with negative values indicating caudal), and $z$ (dorsoventral, with positive values for dorsal) axes in the Talairach space. Individual activations were significant at $p<0.05$, corrected for multiple comparisons within the Rolandic template volume (Fig. 1) unless marked otherwise $\left({ }^{*} p<0.001\right.$, uncorrected). Activations in the group analysis were significant at $p<0.001$, uncorrected unless marked otherwise ${ }^{* *} p<0.01$, uncorrected). Most of these activations survived thresholds of $p<0.05$, corrected for multiple comparisons within the opercular template volume (Fig. 2) on the single-subject level. - , No activation detected.

activate larger cortical areas than natural stimuli (Pratt et al., 1979; Forss et al., 1994). Moreover, when considering the historical experiments, one must bear in mind that the electrostimulation of exposed cortical surface used at the time was barely focal and that the investigators analyzed the patients' verbal descriptions of the sensations that had been evoked. The close proximity of trunk, penis, and leg representations could have resulted in nonprecise reports of sensation in smaller represented body parts, and patients may have also preferred to report leg or trunk sensations even if experiencing genital sensation at the same time. The fact that only $<1 \%$ of the patients that were studied reported distinct genital sensation speaks in favor of these interpretations (Penfield and Rasmussen, 1950). Here we provide evidence that the entire somatotopic sequence is represented continuously on the cortical convexity. Conversely, we found no indication of a penile representation in the mesial wall, even when we used unconventionally low statistical thresholds.

The interpretation that the sensory foci

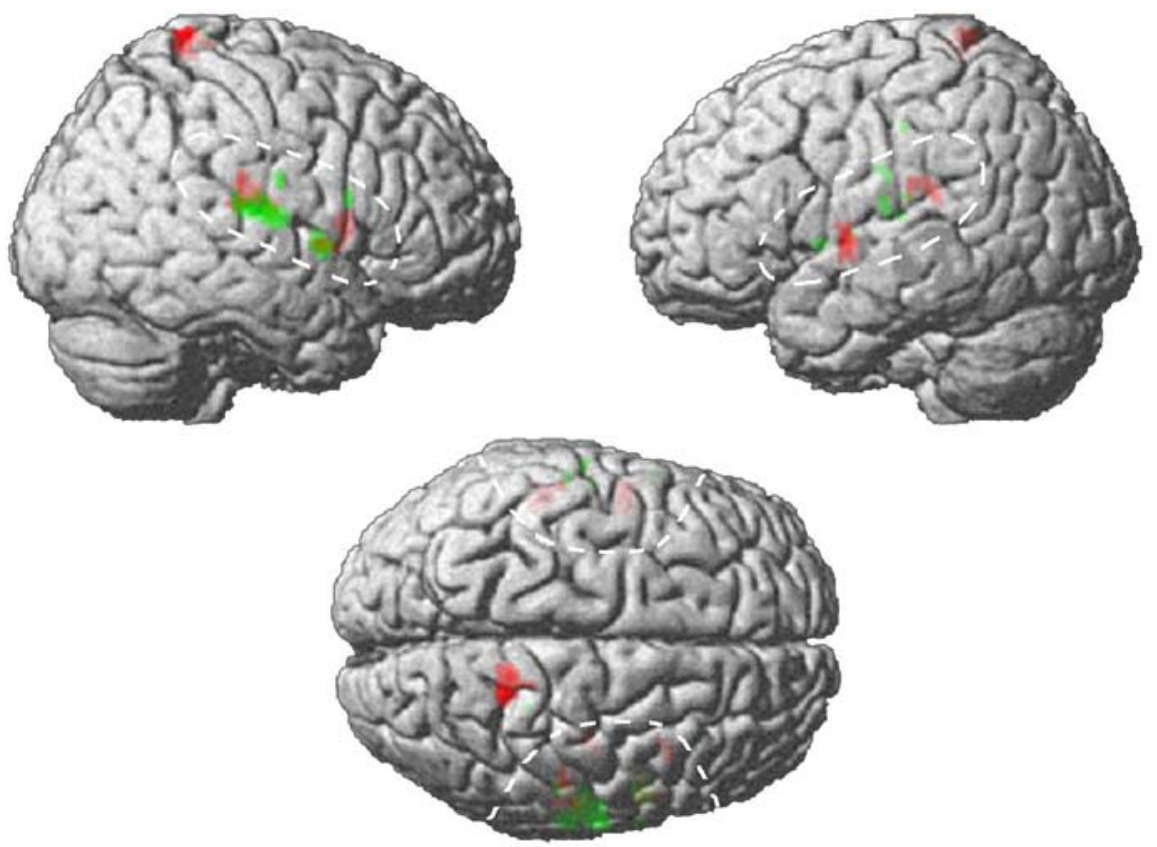

Figure 2. Stimulation of the toe and penis leads to contralateral primary somatosensory and bilateral activation in the opercular secondary somatosensory cortex. Random effects group results were significant at $p<0.001$ (uncorrected), but activations were thresholded at $p<0.006$ for better visibility when projected onto a template-rendered brain. Red and green signify activations related to toe and prepuce. On the single-subject level, most activations reached a significance of $p<0.05$, corrected for multiple comparisons within the delineated opercular template volume (dashed lines). we found on the lateral convexity do indeed belong to the primary sensory cortex is supported by macroscopic anatomical data showing that in the vast majority of humans, the postcentral gyrus does not reach the interhemispheric fissure (Grefkes et al., 2001). On the cytoarchitectonic level, Brodmann area 2 ends on the lateral surface of the postcentral gyrus, and even the probability of Brodmann areas 3 and 1 extending onto the mesial postcentral wall is as low as $30 \%$ (Geyer et al., 1999, 2000; Grefkes et al., 2001; Eickhoff et al., 2005). Hence it cannot be ruled out that in a few individuals, functional responses to foot stimulation might extend into the interhemispheric fissure.
We also used recently developed techniques for relating functional neuroimaging results defined in stereotactic coordinates to cytoarchitectonic areas (Eickhoff et al., 2005). Despite the considerable influence of interindividual variability, which attenuates the certainty with which area labels can be assigned on this basis, we obtained probabilities of $\sim 60 \%$ for our response foci for penis and foot stimulation to arise from Brodmann area $3 \mathrm{~b}$ or Brodmann area 1 . This result, together with that of an orderly somatotopic sequence, is in good agreement with studies on nonhuman primates. In chimpanzees (Woolsey, 1964) and macaques (Rothemund et al., 2002), the genital representation also clearly 


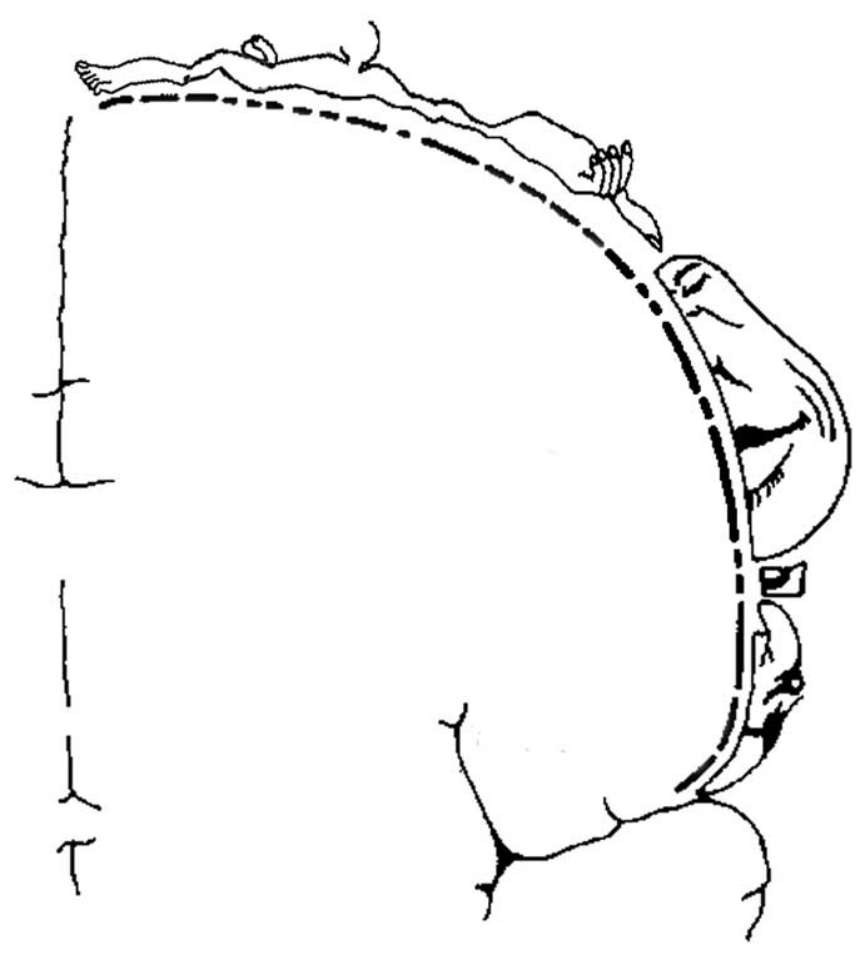

Figure 3. A modified version of Penfield and Rasmussen's sensory homunculus.

lies on the convexity of the hemisphere. In the latter study, the authors localized the sensory focus for the genitals to the border between Brodmann areas 3b and 1, as we did in humans. Therefore, the high homology between macaques and human sensory areas at the histological level (Zilles et al., 1995) is paralleled by an identical somatotopic sequence.

\section{The secondary sensory representation of the penis}

The opercular activations shown in our study parallel recently published data on the secondary somatosensory cortex in man. Young et al. (2004) described four histologically different areas within the parietal operculum, OP1-OP4, that were activated with sensory stimulation. According to their definition, we attribute the two parieto-opercular activations to the areas OP1 and OP4 (Table 1). Our findings are supported by the authors' observation that the foot is represented in the contralateral OP1 and OP4. However, in contrast to the data obtained by Young et al. (2004), we found a similar representation in the ipsilateral parietal operculum. Furthermore, the finding of a bilateral representation in OP1 and OP4 is strengthened by the fact that stimulation of the penis also gives rise to OP1 and OP4 activation bilaterally. A bilateral sequence of secondary sensory foci in the mediolateral axis of the operculum has been reported previously (Ruben et al., 2001), with caudal parts of the body represented more medially compared with more cranial parts, a finding that is in agreement with our results showing a more medial secondary representation of the hallux compared with the penis. However, because the stimulation in our study was restricted to the lower half of the body, our results cannot answer detailed questions of somatotopic organization within these putative secondary somatosensory cortices but rather can only confirm the responsiveness of OP1 and OP4 to cutaneous stimuli.

In conclusion, we have shown that the primary sensory cortical representation of the lower half of the body is confined to the convexity of the hemisphere along the postcentral gyrus and does not extend into the interhemispheric fissure. In particular, the male genital is represented between the legs and the trunk and thus is in accord with the logical somatotopic sequence. Our findings are summarized in a refined version of Penfield and Rasmussen's classical homunculus diagram (Fig. 3).

\section{References}

Allison T, McCarthy G, Luby M, Puce A, Spencer DD (1996) Localization of functional regions of human mesial cortex by somatosensory evoked potential recording and by cortical stimulation. Electroencephalogr Clin Neurophysiol 100:126-140.

Bradley WE, Farrell DF, Ojemann GA (1998) Human cerebrocortical potentials evoked by stimulation of the dorsal nerve of the penis. Somatosens Mot Res 15:118-127.

Eickhoff SB, Stephan KE, Mohlberg H, Grefkes C, Fink GR, Amunts K, Zilles K (2005) A new SPM toolbox for combining probabilistic cytoarchitectonic maps and functional imaging data. NeuroImage 25:1325-1335.

Feinsod M (2005) Kershman's sad reflections on the homunculus: a historical vignette. Neurology 64:524-525.

Foerster O (1936) Sensible corticale Felder. In: Handbuch der Neurologie. Berlin: Springer.

Forss N, Salmelin R, Hari R (1994) Comparison of somatosensory evoked fields to airpuff and electric stimuli. Electroencephalogr Clin Neurophysiol 92:510-517.

Geyer S, Schleicher A, Zilles K (1999) Areas 3a, 3b, and 1 of human primary somatosensory cortex. NeuroImage 10:63-83.

Geyer S, Schormann T, Mohlberg H, Zilles K (2000) Areas 3a, 3b, and 1 of human primary somatosensory cortex. Pt 2. Spatial normalization to standard anatomical space. NeuroImage 11:684-696.

Grefkes C, Geyer S, Schormann T, Roland P, Zilles K (2001) Human somatosensory area 2: observer-independent cytoarchitectonic mapping, inter-individual variability, and population map. NeuroImage 14:617-631.

Makela JP, Illman M, Jousmaki V, Numminen J, Lehecka M, Salenius S, Forss N, Hari R (2003) Dorsal penile nerve stimulation elicits left-hemisphere dominant activation in the second somatosensory cortex. Hum Brain Mapp 18:90-99.

Nakagawa H, Namima T, Aizawa M, Uchi K, Kaiho Y, Yoshikawa K, Orikasa S, Nakasato N (1998) Somatosensory evoked magnetic fields elicited by dorsal penile, posterior tibial and median nerve stimulation. Electroencephalogr Clin Neurophysiol 108:57-61.

Penfield W, Rasmussen T (1950) The cerebral cortex of man. New York: Macmillan.

Pfeifer B (1920) Die Störungen der Sensibilität im Gebiete der GenitoAnalhaut bei Hirnverletzten. Zeitschrift der gesamten Neurologie und Psychiatrie 53:5-38.

Pratt H, Starr A, Amlie RN, Politoske D (1979) Mechanically and electrically evoked somatosensory potentials in normal humans. Neurology 29:1236-1244.

Rothemund Y, Qi HX, Collins CE, Kaas JH (2002) The genitals and gluteal skin are represented lateral to the foot in anterior parietal somatosensory cortex of macaques. Somatosens Mot Res 19:302-315.

Ruben J, Schwiemann J, Deuchert M, Meyer R, Krause T, Curio G, Villringer K, Kurth R, Villringer A (2001) Somatotopic organization of human secondary somatosensory cortex. Cereb Cortex 11:463-473.

Talairach J, Tournoux P (1988) Co-planar stereotaxic atlas of the human brain. Stuttgart, Germany: Thieme.

Woolsey CN (1964) Cortical localization as defined by evoked potential and electrical stimulation studies. In: Cerebral localization and organization (Schaltenbrand G, Woolsey CN, eds), pp 17-26. Madison, WI: University of Wisconsin.

Young JP, Herath P, Eickhoff S, Choi J, Grefkes C, Zilles K, Roland PE (2004) Somatotopy and attentional modulation of the human parietal and opercular regions. J Neurosci 24:5391-5399.

Zilles K, Schlaug G, Matelli M, Luppino G, Schleicher A, Qu M, Dabringhaus A, Seitz R, Roland PE (1995) Mapping of human and macaque sensorimotor areas by integrating architectonic, transmitter receptor, MRI, and PET data. J Anat 187:515-537. 